\title{
Associations between gastric sensorimotor function, depression, somatization, and symptom-based subgroups in functional gastroduodenal disorders: are all symptoms equal?
}

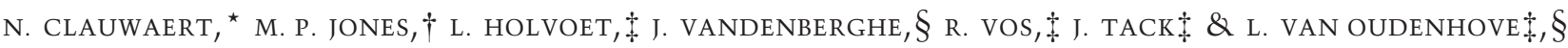

${ }^{\star}$ Child and Adolescent Psychiatry, University Psychiatric Centre, University Hospital Gasthuisberg, University of Leuven, Leuven, Belgium

$\dagger$ Department of Psychology, Macquarie University, Sydney, Australia

†Gastroenterology Section, Department of Pathophysiology, Translational Research Center for Gastrointestinal Disorders (TARGID), University of Leuven, Leuven, Belgium

§Liaison Psychiatry, University Psychiatric Centre, University Hospital Gasthuisberg, University of Leuven, Leuven, Belgium

\begin{abstract}
Background Previous work indicated that psychosocial factors (depression and somatization) are more strongly associated with symptom severity and weight loss in functional dyspepsia (FD) than gastric sensorimotor function. However, there is conflicting evidence regarding the association of these etiopathogenetic factors with Rome III symptombased subgroups in FD [epigastric pain syndrome (EPS), postprandial distress syndrome (PDS)]. We aimed to test whether gastric sensitivity and emptying, depression, and somatization are differentially associated with empirically derived functional gastroduodenal disorders (FGD) symptom factors in one comprehensive model. Methods In 259 tertiary care FD patients, we studied gastric sensorimotor function with barostat and gastric emptying breath test. Depression, somatization, and FGD symptoms were measured using self-report questionnaires. Confirmatory factor analysis (CFA) on 7 FGD symptoms was used to determine the fit of a latent variable structure based on Rome III symptom-based subgroups. Structural equation modeling (SEM) was used to test the
\end{abstract}

Address for Correspondence

Dr. Lukas Van Oudenhove, Translational Research Centre for Gastrointestinal Diseases (TARGID), University Hospital

Gasthuisberg, O\&N1, 7th floor, bus 701, Herestraat 49,

B-3000 Leuven, Belgium.

Tel: +3216330147; fax: +3216345939 ;

e-mail: lukas.vanoudenhove@med.kuleuven.be

Received: 17 March 2012

Accepted for publication: 18 June 2012 putative relationships of the symptom factors with gastric sensorimotor function, depression, and somatization. Key Results The results of the CFA show a good fit $\left[C_{\min } / D F=1.54\right.$, CFI(comparative fit index) $=0.97]$ for the three-factor solution based on Rome III subgroups. The SEM also fitted the data well $\left(C_{\min } / D F=1.24, C F I=0.98\right)$ and demonstrated that gastric sensitivity and depression are associated with PDS and nausea and vomiting. Gastric emptying is uniquely associated with EPS and somatization is strongly associated with all three symptom factors. Conclusions $\leftrightarrow$ Inferences Confirmatory factor analysis confirms the existence of three FGD symptom factors, corresponding to Rome III symptom-based subgroups. The SEM results suggest that different psychobiological mechanisms may play a role in these subgroups.

Keywords depression, functional gastroduodenal disorders, gastric sensorimotor function, somatization, structural equation modeling, symptom-based subgroups.

\section{INTRODUCTION}

A substantial proportion of patients with functional gastrointestinal disorders (FGID) have chronic symptoms that can be attributed to the gastroduodenal region. ${ }^{1}$ According to the Rome III classification, a subdivision of these functional gastroduodenal disorders (FGD) into four categories is proposed: (i) functional dyspepsia (FD), (ii) belching disorders, (iii) nausea 
and vomiting disorders, and (iv) rumination syndrome. The first symptom-based FGD, FD, is one of the most prevalent FGID, and is defined as 'the presence of symptoms thought to originate in the gastroduodenal region, in absence of any organic, systemic, or metabolic disease likely to explain the symptoms ${ }^{\prime}{ }^{1}$ It is a heterogeneous disorder and its pathophysiology is likely to be multifactorial, but remains incompletely understood. ${ }^{2}$ A subdivision of FD into two distinct symptom-based subgroups has been proposed: epigastric pain syndrome (EPS), with epigastric pain and burning as the key symptoms, and postprandial distress syndrome (PDS), with early satiation and postprandial fullness being the defining features. According to the biopsychosocial model, FGD symptoms result from a complex reciprocal interaction between biological, psychological, and social factors. ${ }^{3-5}$ More specifically, three groups of factors are believed to be important in FD. Firstly, FD is associated with gastric sensorimotor dysfunction including hypersensitivity to gastric distension $^{6}$ and delayed gastric emptying, ${ }^{7}$ among others. Secondly, there is growing evidence for an association between FD and both 'trait' (neuroticism, trait anxiety, and alexithymia) and 'state' (anxiety, depression, ...) psychological factors (including psychiatric co-morbidity), not only in treatment-seeking but also in community-based samples. ${ }^{8-13}$ Moreover, high prevalence of (childhood) physical and/or sexual abuse has been reported in $\mathrm{FD}^{14}$ in parallel with earlier findings in studies concerning the irritable bowel syndrome (IBS). ${ }^{15}$ Thirdly, a (psychological) tendency to experience and report multiple somatic symptoms that cannot be (adequately) explained by organic findings (often referred to as 'somatization') is playing a key role in FGID in general and FD in particular. ${ }^{16-19}$ Among these different factors, mainly somatization and, to a lesser extent, depression have been previously found to be more important determinants of overall FD symptom levels than gastric sensorimotor function. ${ }^{2}$ However, regarding the association of the symptom-based subgroups (PDS and EPS) with psychopathology, somatization, and gastric sensorimotor dysfunction, there is conflicting evidence in the literature: an earlier study in outpatients found psychopathology, somatization, and hypersensitivity to be associated with EPS symptoms and delayed gastric emptying with PDS symptoms, ${ }^{17}$ whereas more recent studies, both population based and in outpatients, found that psychopathology is mainly associated with a clinical diagnosis of PDS. ${ }^{20,21}$ This study aims to contribute in resolving this conflict by testing whether gastric sensitivity and emptying (gastric sensorimotor functions), depression, and somatization are differentially associated with empirically derived FGD symptom factors. Depression and somatization were chosen based on their association with overall FGD symptom levels in a previous study by our group. ${ }^{2}$ More specifically, based on previous literature outlined above, we hypothesized that gastric hypersensitivity and delayed gastric emptying would be associated with EPS and PDS symptoms, respectively. Furthermore, we hypothesized that somatization would be associated with all symptom factors, as it is conceptualized as a tendency to report multiple somatic symptoms in general. Finally, we hypothesized that depression would be mainly associated with PDS symptoms.

\section{MATERIALS AND METHODS}

\section{Patient sample}

Consecutive Dutch-speaking patients who recently received a clinical diagnosis of FD according to Rome II criteria (with or without comorbid) functional vomiting [either at their visit to the general GI or GI motility clinic at the University Hospital Leuven (tertiary care) or at a recent secondary care gastroenterologist visit that lead to the referral] were asked to complete an FGD symptom questionnaire, ${ }^{2,6,7,17,22-24}$ and questionnaires on anxiety, depression, and somatization between 2002 and 2009. Patients with rumination syndrome were excluded based on typical elements in the history /occurrence of effortless regurgitation during or immediately after the meal, absence of major nausea, and ability to reswallow the regurgitated material). Patients with aerophagia were also excluded based on the presence of objectively observed air swallowing.

Gastric sensitivity and gastric emptying were tested using barostat and breath test, respectively. The patient samples reported on in the present study do partially overlap with samples from other studies by our group. ${ }^{2,22,24}$ However, the hypotheses tested and analyses performed in the present article are novel and have not been reported on elsewhere. All patients underwent history taking and clinical examination, upper GI endoscopy, routine biochemistry, and abdominal ultrasound. Inclusion criteria were the presence of symptoms for at least 12 weeks during the last 12 months, in the absence of organic, systemic, or metabolic disease. Symptoms had to be present at least 3 days per week, with two or more symptoms scored as relevant or severe on an FGD symptom questionnaire. Exclusion criteria were the presence of esophagitis, gastric atrophy, erosive gastroduodenal lesions on endoscopy, heartburn as a predominant symptom, a history of peptic ulcer, major abdominal surgery, and the use of non-steroidal anti-inflammatory drugs or steroids. The protocol had been approved by the University Hospitals Leuven ethical committee prior to the start of patient recruitment.

\section{Gastric sensorimotor function testing}

Gastric sensitivity Details about gastric sensorimotor function testing have been published recently. ${ }^{24}$ Briefly, we used our standard barostat protocol. During isobaric stepwise distension, patients scored their perception of upper abdominal sensations at the end of every distending step using a graphic rating scale (0-6) with verbal descriptors (0: no sensation; 5: discomfort; 6: pain). Discomfort threshold was defined as the lowest pressure above 
minimal distending pressure (MDP) evoking a perception score of 5 or more.

Emptying for solids Gastric emptying for solids was measured using the previously validated ${ }^{14} \mathrm{C}$ octanoic acid breath test. ${ }^{25}$ Briefly, all studies were performed in the morning after an overnight fast. The test meal consisted of $60 \mathrm{~g}$ of white bread; one egg, the yolk of which was doped with $74 \mathrm{kBq}{ }^{14} \mathrm{C}$ octanoic acid sodium salt; and $300 \mathrm{~mL}$ of water. Breath samples were taken before the meal and at 15-min intervals for a period of $240 \mathrm{~min}$ postprandially. Gastric half emptying time $\left(t^{1 / 2}\right)$ was calculated as previously described. ${ }^{25}$

\section{FGD symptoms}

On the day of this study, the intensity of eight FGD symptoms (fullness, early satiation, bloating, vomiting, nausea, epigastric pain, epigastric burning, and belching) was scored on a Likert scale with a range from absent (0), mild (1), moderate (2), to severe (3), as previously described. ${ }^{23}$ Associations between symptoms and gastric sensorimotor function and between symptoms themselves have been hypothesized earlier for all symptoms except belching. Belching has been included in the initial exploratory factor analysis of symptoms to confirm this hypothesis.

\section{Depression and somatization}

Depression The depression module of the Patient Health Questionnaire (PHQ-9) was used to screen for depressive comorbidity according to the Diagnostic and Statistical Manual of Mental Disorders (DSM-IV). ${ }^{26}$ The nine DSM-IV depressive symptoms (past 2 weeks) are rated on Likert scales (0-3). Depression score (sum of the nine items) was used as a continuous variable.

Somatization The PHQ somatoform disorder module (PHQ-15) is a self-report questionnaire composed of 15 somatic symptoms, including 14 of the 15 most prevalent DSM-IV disorder criteria. ${ }^{28}$ All items are rated on a Likert scale (0-2). The PHQ-15 has high internal reliability and convergent and discriminant validity.

A limitation of the PHQ-15 as a self-report measure (without interview) is that it cannot distinguish between 'medically explained' and 'unexplained' symptoms ${ }^{27}$ which is an important feature of the 'somatization' concept. ${ }^{19,28}$ In this study, adequate clinical and technical investigations were performed to rule out a medical explanation of GI symptoms but not systematically of other somatic symptoms as included in the PHQ-15, although major non-GI medical comorbidity that may account for these somatic symptoms was ruled out on an 'as needed' basis. Nevertheless, it should be emphasized that the PHQ symptom count in this study can only be characterized as indicative of somatization. However, total self-reported PHQ somatic symptom counts are highly associated with physician rated somatoform disorder symptom counts. ${ }^{28,29}$

\section{Statistical analysis}

EFA Exploratory factor analysis (EFA) was performed using SAS software version 9.2 (SAS Institute, Cary, NC, USA). Exploratory factor analysis is a statistical method used to assess correlation among observed variables that may suggest a smaller number of unobserved variables called factors or latent variables. The two main purposes of factor analysis are to reduce the complexity of the data and to explore the theoretical structure among the set of observed variables. The theoretical questions about the underlying structure of complex phenomena can be explored and empirically tested using factor analysis. In this study principal component analysis (PCA) has been used to suggest the optimal factor structure of the eight symptoms, and to check whether this is what would be predicted by the Rome III classification of FD symptoms. Orthogonal varimax rotation has been used as the resulting factors are statistically independent. Factors explaining more than $10 \%$ of the variance were retained. Exploratory factor analysis was performed on eight FGD symptom ratings in the full patient sample. It should be noted, however, that EFA does not formally assess the fit of the resulting factors structure, although calculation of explained variance goes some way toward this. Assessment of fit is achieved more formally through confirmatory factor analysis.

CFA Confirmatory factor analysis (CFA) is used to assess whether a priori hypothesized latent variables in the model are adequately supported by the data ('measurement model'). In CFA, path coefficients between a latent variable and its 'underlying' observed variables should be interpreted in a similar way to factor loadings in EFA. Confirmatory factor analysis was used in the subsample of patients for whom gastric sensorimotor function and psychosocial date were available, to confirm both the a priori hypothesized latent variable structure and the results from EFA in the full patient sample. Moreover, the 'measurement model' obtained by CFA was used in subsequent SEM (see below).

SEM Confirmatory factor analysis and structural equation model (SEM) analyses were performed using MPLUs software version 5 (Muthén \& Muthén, Los Angeles, CA, USA). Significance level was set at $P<0.05$ (two-tailed). Structural equation model is an extension of general linear models (GLM), in which multiple regression equations are estimated simultaneously rather than predicting one single dependent variable from one or more independent variables. There are a range of fit statistics available in SEM with no single statistic fully characterizing the SEM goodness of fit; a good 'fit' indicates that the model reproduces the observed variance-covariance structure in the data well and hence is a plausible model for the relationships between the variables of interest. This does not, however, indicate that the model specified is in some sense a uniquely optimal model although its a priori specification does increase confidence. In the present study, we use $C_{\min } / \mathrm{DF}$ and comparative fit index (CFI) as fit statistics. Values of $C_{\min } / \mathrm{DF}<2.0$ and values of CFI $>0.95$ are generally regarded as indicative of acceptable fit. ${ }^{30}$ A SEM can be viewed as having a measurement component that describes how observed variables group onto latent variables (CFA) and a structural component that describes the relationship between these latent variables and/or between the latent variables and observed variables. Structural equation model has been used to test the fit of the full $a$ priori hypothesized model (measurement and structural components combined) and the statistical significances of the paths specified in the model. Path coefficients in the SEM model should be interpreted in the same way as standardized regression coefficients in GLM.

\section{RESULTS}

\section{Descriptive results}

Two hundred and fifty-nine FD patients $175.3 \%$ women; mean age $39.5 \pm 12.9$ ) participated in this study. The mean gastroduodenal symptoms score was 
$13.4 \pm 4.8$. Means for the eight individual FGD symptoms fell in the mild to moderate range. According to Rome II classification, 21 patients $(8 \%)$ were diagnosed with comorbid functional vomiting.

\section{EFA}

Table 1 shows the results from the EFA on the eight FGD symptoms. According to the results from the factor analysis, the eight FGD symptoms can be grouped into four latent variables or symptom factors. The first factor combines the symptoms 'fullness', 'early satiation', and 'bloating', and corresponds to Rome III subgroup PDS. The second factor combines the symptoms 'vomiting' and 'nausea', corresponding to the Rome III subgroup nausea/vomiting disorders, and the third factor combines 'epigastric pain' and 'epigastric burning', which corresponds to the Rome III subgroup EPS. A fourth factor consists of belching only and corresponds to the Rome III subgroup of belching disorders. Seventy-one percent of the total variation is explained by these four factors.

The observed factor loadings are strong (all $>0.55$, most $>0.70$ ). Also two cross-loadings of lesser magnitude have been found, namely between Factor 2 (nausea and vomiting) and epigastric burning and between Factor 3 (EPS) and bloating. These crossloadings are, however, moderate in magnitude ( 0.4).

\section{Confirmatory factor analysis}

In line with recent studies that establish belching as an esophageal rather than gastroduodenal symptom, ${ }^{31,32}$ belching was not hypothesized a priori to fit into the structural model with gastric sensorimotor function, depression, or somatization. This is supported by the empirical evidence from the EFA and hence it was omitted from the CFA and SEM models.

Table 1 Results of the exploratory factor analysis

\begin{tabular}{lcrrr}
\hline & Factor 1 & Factor 2 & Factor 3 & Factor 4 \\
\hline Fullness & $\mathbf{0 . 8 7}$ & 0.15 & -0.02 & -0.10 \\
Early satiation & $\mathbf{0 . 7 4}$ & 0.22 & 0.08 & 0.01 \\
Bloating & $\mathbf{0 . 6 5}$ & -0.23 & 0.42 & 0.15 \\
Vomiting & 0.11 & $\mathbf{0 . 8 2}$ & -0.06 & 0.01 \\
Nausea & 0.11 & $\mathbf{0 . 7 1}$ & 0.28 & 0.02 \\
Pain & 0.13 & 0.07 & $\mathbf{0 . 8 8}$ & -0.06 \\
Epigastric burning & 0.04 & 0.45 & $\mathbf{0 . 5 6}$ & 0.21 \\
Belching & -0.01 & 0.05 & 0.03 & $\mathbf{0 . 9 8}$ \\
\hline Eigenvalue & 2.38 & 1.33 & 1.07 & 0.91 \\
Proportion explained & $30 \%$ & $17 \%$ & $13 \%$ & $11 \%$ \\
& & & & \\
\hline
\end{tabular}

Factor loadings $>0.50$ are shown in bold.

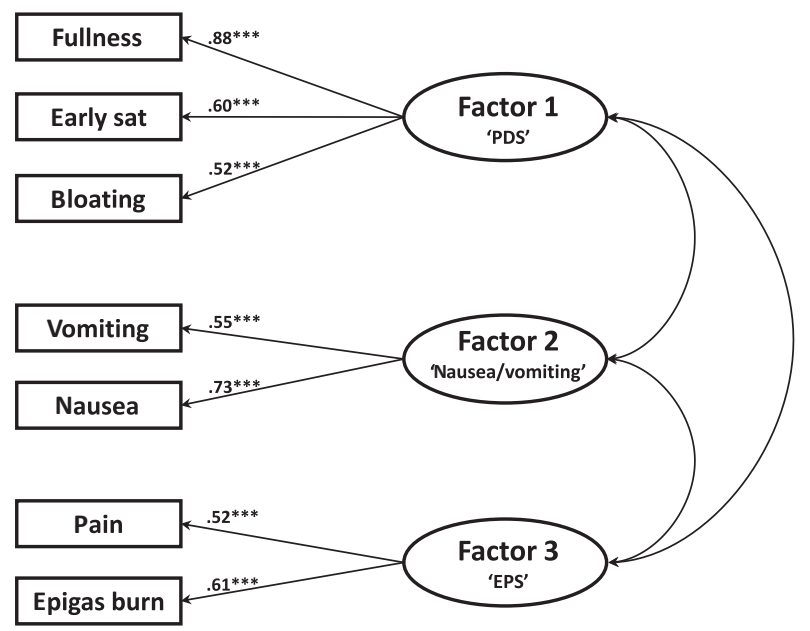

Figure 1 Results of the confirmatory factor analysis. Numbers indicate standardized factor loadings. ${ }^{\star \star \star} P<0.001$; early sat, early satiety; epigas burn, epigastric burning; PDS, postprandial distress syndrome; EPS, epigastric pain syndrome.

Figure 1 shows the results of the CFA, with the three-factor solution based on the EFA and corresponding to Rome III subgroups (EPS, PDS, nausea/vomiting). These results show that the proposed model reveals a good fit $\left(C_{\min } / \mathrm{DF}=1.54, \mathrm{CFI}=0.97\right)$.

\section{SEM}

Structural equation modeling has been used to test the relationship between the symptom factors found in the CFA ('PDS', 'nausea/vomiting', and 'EPS'), on the one hand and gastric discomfort threshold, gastric emptying of solids, depression, and somatization on the other hand. In previous work investigating relations between these factors, ${ }^{17}$ only bivariate correlation analyses have been performed. In the present study, however, all observed data are fitted into a single model (Fig. 2).

The model fits the data well $\left(C_{\min } / \mathrm{DF}=1.24\right.$, $\mathrm{CFI}=0.98$ ). Gastric discomfort threshold is significantly and negatively associated $(-.22)$ with the 'PDS' factor $(P<0.001)$. The association between gastric discomfort threshold and the 'nausea/vomiting' factor is smaller $(-.17)$ but also significant $(P=0.028)$, whereas no association with the 'EPS' factor $(P=0.39)$ was found. Somatization is strongly associated with all three symptom factors $(P<0.001)$, which is an expected finding as somatization is a measure for the tendency to report multiple symptoms. Depression is only significantly related to the 'PDS' factor $(P=0.020)$, to a lesser extent with the 'nausea/vomiting' factor $(P=0.063)$, and not with the 'EPS' factor $(P=0.92)$. Gastric emptying of solids is strongly associated with the 'EPS' factor $(P=0.003)$ and not with the 


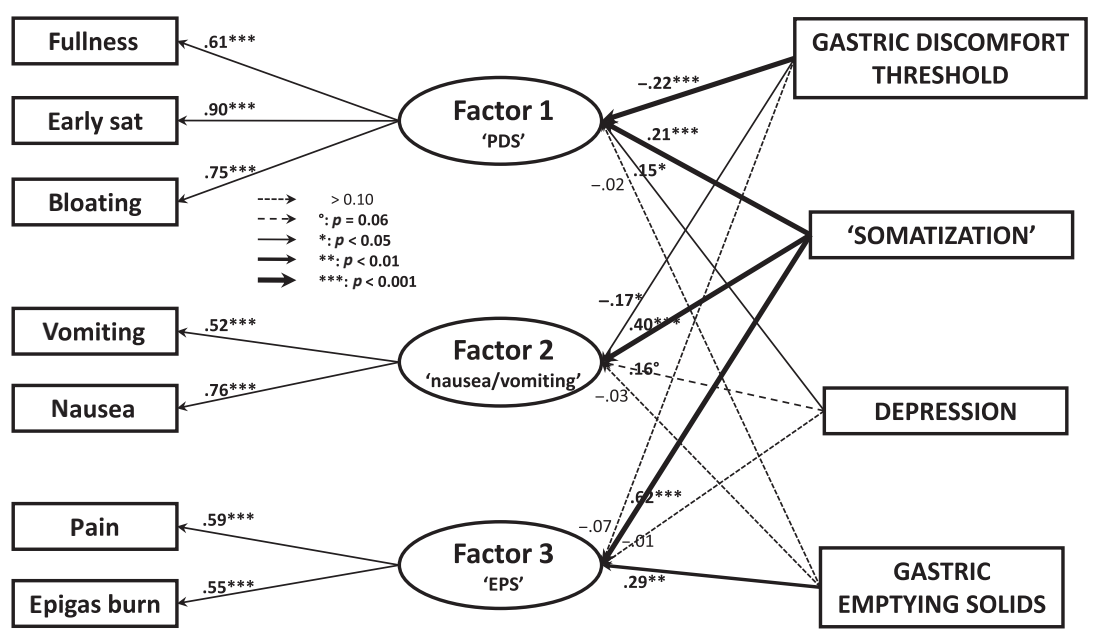

Figure 2 Structural equation model relating the symptom factors with gastric sensorimotor function, depression, and somatization. Numbers indicate standardized factor loadings; early sat, early satiety; epigas burn, epigastric burning; PDS, postprandial distress syndrome; EPS, epigastric pain syndrome.

'nausea/vomiting' and 'PDS' factors $(P=0.82$ and 0.93 , respectively).

\section{DISCUSSION}

EFA demonstrates that FGD symptoms can be grouped into four factors: a first 'PDS' factor consisting of fullness, early satiation, and bloating; a second 'nausea/vomiting' factor, a third 'EPS' factor composed of pain and epigastric burning, and finally 'belching' as a separate fourth factor. Confirmatory factor analysis shows that this factor solution shows a good fit, although we omitted belching from this analysis as we did not have any a priori hypothesis about the relationship between belching and any of the gastric sensorimotor dysfunctions we wanted to test in the structural equation model. This factor structure corresponds to the FGD subgroups as proposed in the Rome III classification. This result is in line with previous evidence, as the Rome III classification has been proposed based on similar studies using cluster- and/ or factor analyses on upper GI symptoms. ${ }^{1,22}$ In previous studies investigating the relations between pathogenetic factors and FD subgroups, mostly bivariate (correlational) statistical analyses have been used to test relations between the observed variables, or at best GLM testing the association between one or more independent variables and one single dependent variable. This is, to the best of our knowledge, the first study investigating these relationships in one single model using SEM. Structural equation model allows us to answer a set of interrelated research questions in a single, systematic, and comprehensive analysis by modeling the relationships among multiple indepen- dent and dependent constructs, whether observed or latent, simultaneously. ${ }^{33}$ Therefore, we believe this study provides new and more comprehensive insights concerning the associations between four relevant pathogenetic mechanisms (gastric sensitivity and emptying, depression, and somatization) and symptom factors or symptom-based subgroups in FGD.

Contrary to our hypothesis, gastric discomfort threshold was found to be strongly associated with the 'PDS' factor and gastric emptying of solids was strongly associated with the 'EPS' factor. In line with our hypothesis, however, somatization was associated with all three factors, more strongly than depression, which was only significantly associated with the 'PDS' factor. As somatization is descriptively defined and measured as a general tendency to report multiple physical complaints, it is conceivable that patients with higher somatization levels also tend to report more FGD symptoms in general, rather than reporting increased levels of some symptoms only. This finding is also in line with a previous finding by our group, showing that somatization is the most important factor associated with overall level of FGD symptoms, as measured by the sum score of all individual FGD symptom items. ${ }^{2}$

The findings from this study are in accordance with other studies. ${ }^{20,21}$ In a population-based study, Aro et $a .^{20}$ found that psychiatric comorbidity is linked to PDS but not to EPS. This is similar to our study in that we also show that PDS rather than EPS symptoms are associated with psychiatric comorbidity, although we found a significant association with depression levels, whereas Aro et al. found that PDS was significantly associated with anxiety but not depression. Somatiza- 
tion was not included in their large population-based study. In the present study, the psychosocial variables used in the model (somatization and depression) were selected based on their association with overall FGD symptom levels in a previous analysis by our group. ${ }^{2}$ However, when adding trait anxiety or current anxiety to the present model, neither of both showed any associations with any of the symptom factors, and adding any of the two anxiety variables did not change the results for the other variables. In an outpatient sample of FD patients, Hsu et al. ${ }^{21}$ found that the diagnosis of PDS was independently associated with somatization, depression, phobia, and additional (psychological) symptoms after adjustment for the diagnosis of EPS, age, gender, educational level, and occupational status. None of these studies included gastric sensorimotor function variables. However, it should be noted that in both these studies, performed after the introduction of Rome III criteria, FD patients were formally diagnosed with EPS and/or PDS. Subsequently, the association between having a diagnosis of EPS or PDS and psychosocial factors was studied and may have been influenced by the large overlap between PDS and EPS. As most of our data have been collected before the introduction of Rome III criteria, such a formal diagnosis was not made in the present study. Therefore, we used factor analytical techniques to empirically derive symptom-based factors which were in line with Rome III classification and used for subsequent association analysis with the etiopathogenetic factors studied.

The findings that gastric discomfort threshold is most strongly related with the 'PDS' and not with the 'EPS' factor and, conversely, that gastric emptying of solids is strongly related with the 'EPS' but not with the 'PDS' factor are at variance with previous findings and with our a priori hypothesis. A previous study by Fischler et al. ${ }^{17}$ based on a different, although similarly recruited Belgian FD patient sample, used similar methods (EFA and CFA) to obtain symptom-based factors that are largely in line with those found in the present study, although early satiety loaded on the nausea/vomiting rather than the bloating/fullness factor in the previous study. Subsequently, bivariate associations between these symptom factors and pathogenetic mechanisms, including gastric sensitivity, emptying, and somatization, were studied. Depression was not included in this study, although it included the personality trait of neuroticism, which is an important risk factor for depression. The results were at variance with our present results, in that neuroticism, somatization, and gastric sensitivity were mainly associated with the 'EPS' factor, whereas gastric emptying was associated with the 'PDS' factor. However, in this study, multiple bivariate association test were performed without correction for multiple testing or holding other factors constant, and the associations found were weak (all correlation coefficients $<0.30$ ), whereas more adequate statistical methods were used in this study, allowing to control for all other associations included in the model when testing any given association. However, when conducting bivariate analyses on the present dataset to assess the relationships between the 'PDS' and 'EPS' factors on the one hand and gastric discomfort threshold and emptying on the other, the results for the 'PDS' factor were similar to our multivariate model (i.e., a significant association with gastric discomfort threshold but not with gastric emptying). For the 'EPS' factor, however, a significant bivariate association with both gastric discomfort threshold and emptying was found, indicating that the only lack of association between gastric discomfort threshold and 'EPS' found in our multivariate model is due to controlling for other important variables. The other associations found here seem to represent discrepancies with previous studies which are not due to differences in statistical modeling. Alternatively, different methods in gastric sensory and motor function testing may account for some of the differences, although this does not apply to previous studies from our group where very similar methods were used.

There are several important limitations of this study that should be addressed. Firstly, we only used selfreport measures, which may be prone to certain forms of bias. Although a structured clinical interview remains the 'gold standard' that is superior to selfreport measures for diagnosing psychiatric comorbidity, we carefully chose self-report measures, most of which have been validated with a structured interview as a comparison. Secondly, this is a cross-sectional study, permitting conclusions about associations but not about causal or temporal relationships between the variables studied. For example, it cannot be determined whether the onset of depressive symptoms precedes the onset of FGD symptoms or vice versa. Moreover, the cross-sectional nature of this study also does not account for likely possibility that subjects prospectively may well exhibit EPS-PDS 'crossover' with time. These results should therefore be interpreted with sufficient caution and require replication in longitudinal studies before any definite conclusions can be drawn. Given the lack of such studies at this moment, however, we feel that these results provide important evidence about the relative contribution of gastric sensorimotor function, depression, and somatization in FGD symptom generation. Thirdly, the 
patient population consists of tertiary care patients, limiting generalizability of the results toward other populations. The gender distribution is high compared to the lack of female predominance in FD reported in the literature, ${ }^{34}$ but in line with previous publications by our group. Moreover, the ratio of women to men is typically higher in tertiary care patients. ${ }^{35}$ As patients were recruited consecutively, they reflect the average tertiary care patients seen in our clinic and were therefore heterogeneous in terms of onset and course of illness, psychiatric comorbidity, previous medication use, etc.

The results from this study might have some potentially important clinical implications. Firstly, this is yet another indication that it is important to systematically check extra-intestinal somatic symptom reporting (i.e., somatization) in patients consulting the gastrointestinal division with unexplained symptoms thought to originate from the gastroduodenal region. This conclusion can be made on the base of the strong association between all three FGD symptom factors and somatization. Furthermore, the present results seem to suggest that routine investigation of psychiatric symptoms, particularly depression, may be even more important in PDS compared with EPS patients, although we believe it is crucial to investigate psychiatric comorbidity in all FGID patients. This is an important conclusion as it has been shown in IBS that patients with psychiatric comorbidity or high levels of somatization are less responsive to standard treatment aimed at GI symptoms, and benefit more from treatments targeting 'central' mechanisms (antidepressants, psychotherapy). ${ }^{36,37}$ A second potentially relevant clinical implication, although somewhat preliminary, concerns the choice of drug treatment based on FGD symptom pattern. When nowadays in clinical practice patients are found to suffer primarily from EPS symptoms, it is common practice to prescribe medications, although to decrease the sensitivity of the stomach, such as tricyclic agents, whereas prokinetics are commonly prescribed for PDS symptoms. This choice of medication is based on the previously shown relations between gastric discomfort threshold with EPS and gastric emptying with PDS symptoms. However, based on the findings in this study, using the most advanced statistical technique that allows to control for all other variables in the model when determining the strength of the association, this strategy should be at least questioned and re-evaluated, although the present results obviously need to be confirmed in other samples. Therefore, it may be premature to draw conclusions regarding the choice of drug treatment from this study alone before such confirmation is available.

Finally, it can be concluded that factor analytical techniques confirm the existence of three FGD symptom factors, corresponding to the Rome III classification. Using SEM, we demonstrated that gastric sensitivity and comorbid depression are associated with 'PDS' and 'nausea and vomiting' symptom factors. Gastric emptying is uniquely associated with the 'EPS' symptom factor and somatization is strongly associated with all three symptom factors. These results suggest that different psychobiological mechanisms may play a role in these different subgroups.

\section{ACKNOWLEDGMENTS}

Dr. Van Oudenhove is a Postdoctoral Research Fellow of the Research Foundation-Flanders (FWO-Vlaanderen).

\section{FUNDING}

This study was funded with a grant from the Research Foundation-Flanders and a Methusalem grant from the University of Leuven to Prof. Tack.

\section{DISCLOSURE}

No competing interests to be declared.

\section{AUTHOR CONTRIBUTION}

$\mathrm{NC}$ and MJ contributed to data analysis and writing of this article; JVDB contributed to study design and data collection; RV and LH contributed to patient recruitment and data collection; IT contributed to study design, patient recruitment, and writing of the manuscript; LVO contributed to study design, data analysis, and writing of the manuscript.

\section{REFERENCES}

1 Tack J, Talley NJ, Camilleri $M$ et al. Functional gastroduodenal disorders. Gastroenterology 2006; 130: 1466-79.

2 Van Oudenhove L, Vandenberghe J, Geeraerts B et al. Determinants of symptoms in functional dyspepsia: gastric sensorimotor function, psy- chosocial factors or somatization? Gut 2008; 57: 1666-73.

3 Engel GL. The need for a new medical model: a challenge for biomedicine. Science 1977; 196: 129-36.

4 Drossman DA. The functional gastrointestinal disorders and the ROME III process. Gastroenterology 2006; 130: 1377-90.
5 Van Oudenhove L, Aziz Q. Recent insights on central processing and psychological processes in functional gastrointestinal disorders. Dig Liver Dis 2009; 41: 781-7.

6 Tack J, Caenepeel P, Fischler B, Piessevaux H, Janssens J. Symptoms associated with hypersensitivity to gastric distention in functional 
dyspepsia. Gastroenterology 2001; 121: 526-35.

7 Sarnelli G, Caenepeel P, Geypens B, Janssens J, Tack J. Symptoms associated with impaired gastric emptying of solids and liquids in functional dyspepsia. Am J Gastroenterol 2003; 98: 783-8.

8 Locke R, Weaver AL, Joseph Melton L, Talley NJ. Psychosocial factors are linked to functional gastrointestinal disorders: a population based nested case-control study. Am I Gastroenterol 2004; 99: 350-7.

9 Cheng C. Seeking medical consultation. Perceptual and behavioral characteristics distinguishing consulters and non-consulters with functional dyspepsia. Psychosom Med 2000; 62: 844-52.

10 Castillo EJ, Camilleri M, Locke GR et al. A community-based, controlled study of the epidemiology and pathophysiology of dyspepsia. Clin Gastroenterol Hepatol 2004; 2: 985 96.

11 Porcelli P, Taylor GJ, Bagby RM, De Carne M. Alexithymia and functional gastrointestinal disorders. Psychother Psychosom 1999; 68: 263-9.

12 Koloski NA, Talley NJ, Boyce PM. Epidemiology and health care seeking in the functional gi disorders: a population-based study. Am J Gastroenterol 2002; 97: 2290-9.

13 Kindt S, Van Oudenhove L, Mispelon L, Caenepeel P, Arts J, Tack J. Longitudinal and cross-sectional factors associated with long-term clinical course in functional dyspepsia: a 5-year follow-up study. Am I Gastroenterol 2011; 106: 340-8.

14 Geeraerts B, Van Oudenhove L, Fischler B et al. Influence of abuse history on gastric sensorimotor function in functional dyspepsia. Neurogastroenterol Motil 2009; 21: 33-41.

15 Drossman DA. Abuse, trauma, and GI illness: is there a link? Am I Gastroenterol 2011; 106: 14-25.

16 Wilhelmsen I. Somatization, sensitization, and functional dyspepsia. Scand I Psychol 2002; 43: 177-80.

17 Fischler B, Tack J, De Gucht V et al. Heterogeneity of symptom pattern, psychosocial factors, and pathophysiological mechanisms in severe functional dyspepsia. Gastroenterology 2003; 124: 903-10.

18 Naliboff BD. Towards a nondualistic approach to multisystem illness. Am J Gastroenterol 2007; 102: 2777-80.

19 De Gucht V, Fischler B. Somatization. A critical review of conceptual and methodological issues. Psychosomatics 2002; 43: 1-9.

20 Aro P, Talley Nicholas J, Ronkainen J et al. Anxiety is associated with uninvestigated and functional dyspepsia (Rome III criteria) in a Swedish Population-Based Study. Gastroenterology 2009; 137: 94-100.

21 Hsu Y-C, Liou J-M, Liao S-C et al. Psychopathology and Personality trait in subgroups of functional dyspepsia based on Rome III criteria. Am I Gastroenterol 2009; 104: 2534-42.

22 Van Oudenhove L, Holvoet L, Vandenberghe J, Vos R, Tack J. Do we have an alternative for the Rome III gastroduodenal symptom-based subgroups in functional gastroduodenal disorders? A cluster analysis approach. Neurogastroenterol Motil 2011; 23: 730-8.

23 Cuomo R, Sarnelli G, Grasso R et al. Functional dyspepsia symptoms, gastric emptying and satiety provocative test: analysis of relationships. Scand $J$ Gastroenterol 2001; 36: 1030-6.

24 Van Oudenhove L, Vandenberghe J, Geeraerts B et al. Relationship between anxiety and gastric sensorimotor function in functional dyspepsia. Psychosom Med 2007; 69: 455-63.

25 Ghoos YF, Maes BD, Geypens BJ, Hiele MI, Rutgeerts PJ, Vantrappen G. Measurement of gastric emptying rate of solids by means of a carbon labeled octanoic acid breath test. Gastroenterology 1993; 104: 1640-7.

26 Kroenke K, Spitzer RL, Williams JBW. The PHQ-9. Validity of a brief depression severity measure. Int $I$ Gen Med 2001; 16: 606-13.

27 American Psychiatric Association. Diagnostic and Statistical Manual of Mental Disorders, 4th edn. Washington, DC: American Psychiatric Association, 1994.
28 Kroenke K, Spitzer RL, Williams JBW. The PHQ-15: validity of a new measure for evaluating the severity of somatic symptoms. Psychosom Med 2002; 64: 258-66.

29 Kroenke K, Spitzer RL, deGruy FV III et al. A symptom checklist to screen for somatoform disorders in primary care. Psychosomatics 1998; 39: 26372.

30 Schermelleh-Engel K, Moosbrugger $\mathrm{H}$. Evaluating the fit of structural equation models: tests of significance and descriptive goodness-of-fit measures. Methods Psychol Res Online 2003; 8: 23-74.

31 Blondeau K, Boecxstaens V, Rommel $\mathrm{N}$ et al. Baclofen improves symptoms and reduces postprandial flow events in patients with rumination and supragastric belching. Clin Gastroenterol Hepatol 2012; 10: 379-84.

32 Bredenoord AJ, Weusten BL, Sifrim D, Timmer R, Smout AJ. Aerophagia, gastric, and supragastric belching: a study using intraluminal electrical impedance monitoring. Gut 2004; 53: 1561-5.

33 Gefen D, Straub DW, Boudreau M-C. Structural equation modeling and regression: guidelines for research practice. Comm Assoc Inform Syst 2000; 4: 2-77.

34 Chang L, Toner BB, Fukudo S et al. Gender, age, society, culture, and the patient's perspective in the functional gastrointestinal disorders. Gastroenterology 2006; 130: 1435-46.

35 Ouyang A, Wrzos HF. Contribution of gender to pathophysiology and clinical presentation of IBS: should management be different in women? Am I Gastroenterol 2006; 101: S602-9.

36 Creed F, Guthrie E, Ratcliffe J et al. Does psychological treatment help only those patients with severe irritable bowel syndrome who also have concurrent psychiatric disorder? Aust N Z J Psychiatry 2005; 39: 807-15.

37 Creed F, Tomenson B, Guthrie E et al. The relationship between somatisation and outcome in patients with severe irritable bowel syndrome. I Psychosom Res 2008; 64: 613-20. 